\title{
Liga NiCrSiBC: Microestrutura e Dureza de Revestimentos Processados a Arco e a Laser
}

\author{
Leandro J. da Silva ${ }^{1}$, Ana Sofia C. M. D'Oliveira ${ }^{1}$ \\ 1 Laboratório de Engenharia de Superfícies, Departamento de Engenharia Mecânica, Universidade Federal do Paraná - UFPR, \\ Curitiba, PR, Brasil.
}

Recebido: 21 Jan., 2015

Aceito: 13 Mar., 2015

E-mails:

leandro.tools@gmail.com (LJS), sofmat@ufpr.br (ASCMDO)
Este é um artigo publicado em acesso aberto (Open Access) sob a licença Creative Commons Attribution Non-Commercia que permite uso, distribuição e reproduçăo em qualq'uer meio, sem restriçōes desde que sem fins comerciais e que 0 trabalho original seja corretamente citado.
Resumo: A técnica de processamento é determinante sobre características de um revestimento. Isso porque o aporte térmico, que depende da técnica e dos parâmetros, tem influência sobre a diluição e a taxa de solidificação. Em ligas com baixa complexidade metalúrgica, o impacto do processamento com técnicas de deposição que impõem maior taxa de resfriamento podem se traduzir em refino da microestrutura. Espera-se que quanto mais refinada a microestrutura maior a resistência mecânica do revestimento. Entretanto, na deposição de ligas de maior complexidade metalúrgica isso nem sempre ocorre, porque a elevada taxa de resfriamento pode suprimir formação/precipitação de fases responsáveis pela resistência. Neste estudo, a influência do processamento sobre microestrutura e dureza de revestimentos da liga Colmonoy- $6^{\circledR}$ foi avaliada. A liga foi processada por plasma com arco transferido (PTA) e laser de diodo de alta potência (HPDL) sobre chapas de AISI 304 com dois níveis de diluição. Em ambos os casos, revestimentos de boa qualidade e livres defeitos foram obtidos. O aumento do teor de Fe (diluição) e as diferentes taxas de resfriamento decorrem do processamento com diferentes parâmetros e técnicas. Em consequência, ocorrem mudanças significativas na microestrutura e na dureza dos revestimentos que estão associadas à distribuição, morfologia e composição química dos carbonetos e, principalmente, dos boretos.

Palavras-chave: Revestimentos soldado; Processamento; PTA; Laser cladding.

\section{NiCrSiBC Coating: Microstructure and Hardness of Coatings Processed by Arc and Laser}

\begin{abstract}
The processing technique rules the characteristics of coatings. The heat input that depends on the technique and deposition parameters, accounts for changes on the dilution and solidification rate. Depositing alloys with low metallurgical complexity with a faster solidification rate can result on microstructure refining and therefore a higher mechanical resistance is expected. However, in the deposition of more complex alloys, a higher cooling rate can suppress the formation/precipitation of hard phases/compounds compromising the mechanical resistance of coatings. In this study, the influence of the processing technique on microstructure and hardness of Colmonoy- $6^{\circledR}$ alloy coating was analyzed. Coatings were processed by plasma transferred arc (PTA) and high power diode laser (HPDL) on plates of AISI 304 with two different dilutions each. High quality and defect-free coating were processed. Iron content in coatings and their cooling rate were determined by the deposition technique. Consequently, significant changes in microstructure and hardness of coating occurred and were associated with the phase distribution, morphology and size of compounds, mainly of borides.
\end{abstract}

Key-words: Hardfacing; Processing; Plasma Transferred Arc; Laser cladding.

\section{Introdução}

À liga Colmonoy- $6{ }^{\circledR}$ pertence à família de ligas $\mathrm{NiCrSiBC}$, que tem como característica principal à elevada resistência ao desgaste à corrosão em elevadas temperaturas. Essa liga foi originalmente desenvolvida para deposição por aspersão térmica, mas devido as suas excelentes características e menor custo, em relação às superligas de Co, vem sendo depositada por vários processos de soldagem, como PTA [1], TIG [2] e Laser Cladding [3].

Um aspecto importante na deposição de revestimentos soldados é a diluição, isto é, quanto do material de adição se mistura com o substrato [4]. Enquanto uma diluição 
mínima é necessária para garantir a ligação metalúrgica entre o substrato e o revestimento, uma diluição excessiva é indesejável, por promover alterações na composição química e, consequentemente, na microestrutura e no desempenho do revestimento.

Utilizando técnicas diversas, alguns pesquisadores chamaram a atenção para o impacto da diluição sobre as características dos revestimentos NiCrSiBC. Hemmati et al. [3] depositaram a liga Colmonoy- $6{ }^{\circledR}$ com a técnica laser cladding sobre chapas de aço baixo carbono e observaram alterações significativas na microestrutura e redução da dureza dos revestimentos com o aumento da diluição. Os autores atribuem essas alterações ao aumento do teor de Fe e explicam que o Fe modifica e suprime a formação dos boretos do eutético NiSiB, durante a solidificação.

Ramasubbu et al. [2], com objetivo de avaliar o efeito da diluição na microestrutura e dureza da liga Colmonoy-6 ${ }^{\circledR}$, realizaram sobreposição de cinco cordões com o processo TIG, iniciando sobre uma chapa de AISI 316, e observaram que a quantidade de boretos no $1^{\circ}$ e $2^{\circ}$ (maior diluição) cordão é inferior a quantidade no $3^{\circ}, 4^{\circ}$ e $5^{\circ}$ cordão (menor diluição), porém, a quantidade de carbonetos não alterou significantemente. Sugerindo que, os boretos são mais sensíveis à diluição que os carbonetos. Além disso, também concluíram que com o aumento do teor de $\mathrm{Fe}$, não apenas a quantidade, mas morfologia e composição química dos boretos é alterada, no entanto o papel do Fe nessas alterações ainda não está bem compreendido.

Outra abordagem, com a mesma metodologia de Ramasubbu et al. [2], porém com o objetivo de avaliar o efeito da diluição na resistência a corrosão aquosa foi realizada por Chandran e Vinayak [5]. Os resultados apresentados mostram que a resistência à corrosão é menor no $1^{\circ}$ e $2^{\circ}$ cordão. A maior resistência à corrosão ocorreu nos cordões com maior fração volumétrica de boretos $\left(3^{\circ}, 4^{\circ} \mathrm{e} 5^{\circ}\right.$ cordão). Com isso os autores concluíram que a presença de boretos é importante, não apenas para a resistência ao desgaste, mas também para transmitir maior resistência a corrosão ao revestimento.

Uma comparação entre as ligas Colmonoy $-5^{\circledR}$ e Colmonoy- $6^{\circledR}$ com diferentes níveis de diluição foi realizada por Das et al. [6]. A metodologia é semelhante a de Ramasubbu et al. [2], porém, com apenas duas camadas de sobreposição, ou seja, o primeiro cordão sobre o substrato (AISI 316) e o segundo sobre o primeiro. As ligas exibem alterações significativas na microestrutura entre si e em função do nível de diluição. Este resultado vem novamente reforçar a grande sensibilidade da microestrutura das ligas $\mathrm{NiCrSiBC}$ a pequenas alterações na composição química.

A liga Colmonoy- $6^{\circledR}$ também apresenta alta sensibilidade às condições de resfriamento. Ramasubbu et al. [2] e Chandran e Vinayak [5] levantaram essa hipótese quando compararam a dureza do revestimento $\left(5^{\circ}\right.$ cordão com pouca diluição) com a dureza de uma amostra sem diluição, depositada sobre uma chapa de cobre. Ao contrário do esperado, a amostra depositada sobre o cobre, sem diluição e com maior taxa de resfriamento (cobre tem alta condutividade térmica) apresentou menor dureza. Hemmati et al. [7], comprovaram essa hipótese, através de um ensaio de analise térmica diferencial com diferentes taxas de resfriamento e concluíram que para mesma composição química, elevadas taxas de resfriamento suprimiram a formação dos boretos.

De forma geral, nos trabalhos apresentados na literatura, os revestimentos NiCrSiBC foram processados por processos a arco (TIG e PTA) e a Laser (laser cladding). Na comparação entre trabalhos são identificadas alterações significativas na microestrutura dos revestimentos em função das diferentes técnicas de processamento utilizadas, que por sua vez, determinaram o nível de diluição e a taxa de resfriamento. Entretanto, as informações da literatura não esclarecem o impacto do processamento sobre a microestrutura havendo a necessidade de um estudo comparativo sistemático entre as características desses revestimentos depositados por processos a arco e a laser. É neste contexto que o presente estudo se insere com o objetivo de avaliar revestimentos NiCrSiBC processados por PTA e HPDL sobre substratos de AISI 304, quanto a microestrutura desenvolvida em função do nível de diluições impostos.

\section{Materiais e Métodos}

Os principais equipamentos utilizados nesta pesquisa estão listados na Tabela 1. Para a deposição dos cordões foi utilizada a liga $\mathrm{NiCrSiBC}$, comercialmente conhecida como Colmonoy- $6^{\circledR}$. Fornecida na forma de pós-atomizados, com granulometria entre 60-180 $\mu \mathrm{m}$. Como substrato foram utilizadas chapas de aço inoxidável AISI 304 com dimensões de $100 \times 100 \times 12,7 \mathrm{~mm}^{3}$. Na Tabela 2 são apresentadas as composições químicas do material de adição e do substrato. 


\subsection{Deposição dos revestimentos}

Inicialmente, as placas de teste (AISI 304), com dimensões de $101,6 \times 60 \times 12,7 \mathrm{~mm}^{3}$, foram preparadas, para as deposições, através de jateamento abrasivo em ambos os lados (procedimento padrão para remoção de sujeiras e óxidos). 0 material de adição (liga atomizada) foi seco em uma estufa a $100^{\circ} \mathrm{C}$ durante 2 horas. Quanto ao equipamento PTA, trata se de uma fonte de corrente constante, que opera numa faixa de corrente entre 40 e 300 A, equipada com uma tocha com diâmetro do bocal de $3,2 \mathrm{~mm}$, recuo do eletrodo de $3 \mathrm{~mm}$, eletrodo de tungstênio com $3,2 \mathrm{~mm}$ de diâmetro e ângulo de afiação de $60^{\circ}$. Para a deposição com HPDL foi utilizada uma fonte de laser de diodo com potência máxima de $10 \mathrm{~kW}$.

Os revestimentos foram processados utilizando argônio nos três sistemas de gás (gás de proteção, plasma e arraste). Os parâmetros de processamento por PTA estão apresentados na Tabela 3 e por HPDL na Tabela 4. As condições de processamento utilizadas, em ambos os processos, foram estimadas com base na literatura e ajustadas através da realização de testes preliminares, visando à deposição de cordões largos, com bom acabamento e livres de trincas. A variação da diluição foi realizada através da sobreposição de passes, ou seja, o primeiro cordão foi depositado sobre o substrato, e o segundo cordão foi depositado sobre o primeiro.

\subsection{Caracterização}

\subsubsection{Microscopia Eletrônica de Varredura (MEV) e Espectroscopia de Energia Dispersiva (EDS)}

Inicialmente foi realizada preparação metalográfica pelo procedimento convencional. As análises foram realizadas com a amostra sem ataque químico e com o detector de elétrons retroespalhados (BSE). O detector BSE é uma ferramenta muito útil para o estudo devido à habilidade de gerar contraste com base no número atômico, que

Tabela 1. Principais equipamentos utilizados no desenvolvimento do trabalho.

\begin{tabular}{ccc}
\hline Descrição & Modelo & Fabricante \\
Processamento PTA & PTA STARWELD 300 & Deloro \\
Tocha & Excalibur & Kennametal \\
Laser Clading & HighLight D - Series & Coherent \\
MEV & Tescan & Oxford \\
Difração de raios-X & XRD-7000 & Shimadzu \\
Fluorescência de raios-X & EDX-800HS & Shimadzu \\
Dureza & HMV - 2 & Shimadzu \\
\hline
\end{tabular}

Tabela 2. Composição química nominal do substrato e do revestimento.

\begin{tabular}{cccccccccc}
\hline & $\mathbf{C}$ & Si & Mn & $\mathbf{P}$ & $\mathbf{S}$ & $\mathbf{C r}$ & $\mathbf{B}$ & $\mathbf{N i}$ & $\mathbf{F e}$ \\
$\mathrm{NiCrBSiC}$ & 0,7 & $2-4,5$ & - & - & - & 13,5 & $3,1-3,5$ & Base & 4 \\
$\mathrm{AISI} 304$ & $0,04-0,1$ & 0,75 & 2 & 0,04 & 0,03 & 18 & - & $8-10$ & Base \\
\hline
\end{tabular}

Tabela 3. Parâmetros utilizados na deposição por PTA.

\begin{tabular}{ccccccc}
\hline $\begin{array}{c}\text { Distância } \\
\text { tocha/peça }\end{array}$ & $\begin{array}{c}\text { Taxa de } \\
\text { alimentação }\end{array}$ & $\begin{array}{c}\text { Vazão do gás } \\
\text { de plasma }\end{array}$ & $\begin{array}{c}\text { Vazão gás de } \\
\text { arraste }\end{array}$ & $\begin{array}{c}\text { Vazão do gás } \\
\text { de proteção }\end{array}$ & $\begin{array}{c}\text { Velocidade de } \\
\text { avanço }\end{array}$ & $\begin{array}{c}\text { Corrente de } \\
\text { soldagem }\end{array}$ \\
$(\mathrm{mm})$ & $(\mathrm{g} / \mathrm{min})$ & $(\mathrm{L} / \mathrm{min})$ & $(\mathrm{L} / \mathrm{min})$ & $(\mathrm{L} / \mathrm{min})$ & $(\mathrm{mm} / \mathrm{min})$ & $(\mathrm{A})$ \\
10 & 17 & 2 & 0,8 & 15 & 60 & 180 \\
\hline
\end{tabular}

Tabela 4. Parâmetros utilizados na deposição por HPDL.

\begin{tabular}{ccccccc}
\hline $\begin{array}{c}\text { Distância } \\
\text { tocha/peça }\end{array}$ & $\begin{array}{c}\text { Taxa de } \\
\text { alimentação }\end{array}$ & $\begin{array}{c}\text { Vazão do gás } \\
\text { de proteção }\end{array}$ & $\begin{array}{c}\text { Velocidade de } \\
\text { avanço }\end{array}$ & Potência & $\begin{array}{c}\text { Área de } \\
\text { incidência }\end{array}$ & $\begin{array}{c}\text { Densidade de } \\
\text { energia }\end{array}$ \\
$(\mathrm{mm})$ & $(\mathrm{g} / \mathrm{min})$ & $(\mathrm{L} / \mathrm{min})$ & $(\mathrm{mm} / \mathrm{min})$ & $(\mathrm{kW})$ & $\left(\mathrm{mm}^{2}\right)$ & $\left(\mathrm{kW} / \mathrm{mm}^{2}\right)$ \\
50 & Constante & 15 & 60 & 5000 & $16 \times 3$ & 125 \\
\hline
\end{tabular}


torna muito simples distinguir boretos e carbonetos. Também foram realizadas microanálises semi-quantitativas da composição química, mapa e pontual, por EDS. Para microanálise, por EDS, dos boretos e carbonetos foi utilizada uma baixa aceleração dos elétrons $(5 \mathrm{kV})$ para aumentar a sensitividade do sinal do equipamento.

\subsubsection{Dureza}

Para medição da dureza foi utilizado um penetrador Vickers e força normal de $1 \mathrm{kgf}$. Foram realizados 25 ensaios de dureza no topo dos revestimentos. Os valores apresentados representam valores médios das medidas feitas para cada condição de processamento.

\subsubsection{Difração de Raios-X (DRX)}

A identificação das fases nos revestimentos foi realizada através de DRX, numa superfície plana, no topo do revestimento. Na Tabela 5 estão apresentados os parâmetros utilizados no DRX.

\subsubsection{Diluição}

A diluição foi determinada pelo teor de Fe no revestimento, com base nos resultados do EDX (fluorescência de raios $X)$, tendo como referência a composição química do pó. A Equação 1 foi utilizada nos cálculos, onde: Xr é a porcentagem em peso de Fe no revestimento; $X s$ é a porcentagem de Fe no substrato; e Xa é a porcentagem de Fe no material de adição.

$$
\operatorname{Diluição(\% )}=\frac{X r-X a}{X s-X a} * 100
$$

\section{Resultados e Discussão}

Os revestimentos processados por PTA e HPDL, com 1 e 2 cordões apresentam bom acabamento e ausência de defeitos, como trincas, porosidades ou falta de fusão. Como esperado, os maiores níveis de diluição foram encontrados nos revestimentos processados por PTA (Figura 1). Esse resultado é atribuído ao maior aporte de calor deste processo. Entretanto, é difícil fazer uma comparação entre o aporte de energia do PTA e do HDPL, pois os fenômenos envolvidos na geração de calor e as perdas de calor para o ambiente são diferentes. Mas, através da otimização dos parâmetros de processamento é possível obter, para o PTA, níveis de diluição tão baixos quanto o do HPDL. Contudo, deve-se ressaltar que quanto menor a diluição, maior a probabilidade de ocorrer defeitos como falta de fusão, principalmente nos processos a arco, em que a distribuição de energia segue, aproximadamente,

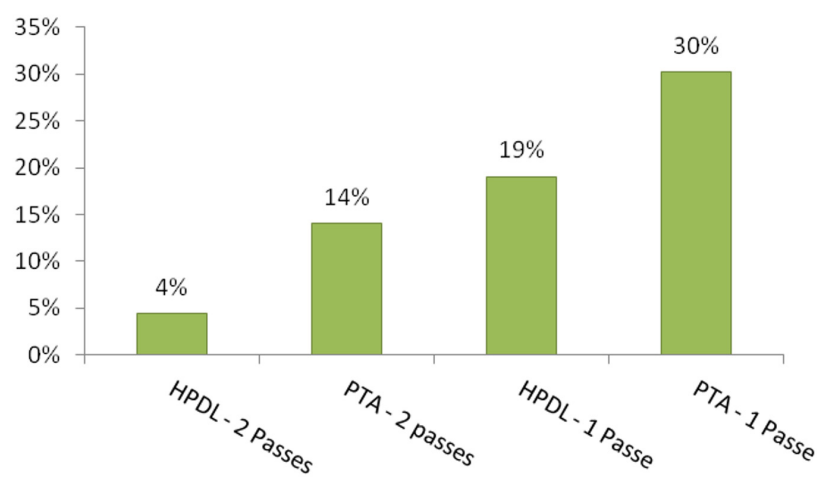

Figura 1. Níveis de diluição em função do processo e do número de sobreposições dos cordões.

Tabela 5. Parâmetros utilizados nos ensaios de DRX.

\begin{tabular}{|c|c|c|c|}
\hline Alvo & Cu K $\alpha(\lambda=0,15406 \mathrm{~nm})$ & Faixa de varredura & $30,0^{\circ}-100^{\circ}$ \\
\hline Tensão & $40 \mathrm{Kv}$ & Velocidade de varredura & 1,0 grau. $\min ^{-1}$ \\
\hline Corrente & $20,0 \mathrm{~mA}$ & Amostragem & $0,02^{\circ}$ \\
\hline Movimentação & $\theta-2 \theta$ & Modo de varredura & Contínua \\
\hline
\end{tabular}


um perfil gaussiano. Enquanto no HPDL a distribuição segue um perfil, aproximadamente, retangular, permitindo processar revestimentos com baixos níveis de diluição e probabilidade de defeitos.

Em consequência dos diferentes níveis de diluição ocorreram alterações significativas na composição química dos revestimentos em relação a liga original, Figure 2, principalmente, nos teores de Fe e Ni. Com o aumento da diluição, o teor de Fe aumenta, aproximadamente, na mesma proporção que o Ni diminui, como esperado pela grande solubilidade entre esses elementos. Não foram medidas alterações significativas nos teores de $\mathrm{Cr}$ e $\mathrm{Si}$, porque os teores desses elementos, no material de adição e no substrato, são semelhantes. Entretanto, é uma pequena variação no teor de $\mathrm{Cr}$ que permite justificar a também pequena variação na fração volumétrica de carbonetos de Cr reportada por Ramasubbu et al. [2].

A variação na composição química dos revestimentos pode ser associada as alterações observadas na microestrutura dos revestimentos. Diversos boretos $\left(\mathrm{CrB}, \mathrm{Ni}_{4} \mathrm{~B}_{3}, \mathrm{Cr}_{5} \mathrm{~B}_{3}, \mathrm{Ni}_{3} \mathrm{~B}\right.$ e $\left.\mathrm{Ni}_{2} \mathrm{~B}\right)$, identificados através de $\mathrm{DRX}$, nos revestimentos com baixa diluição não foram observados nos revestimentos com elevado nível de diluição, Figura 3. Esse comportamento confirmou os resultados reportados por alguns pesquisadores [1-3,5,6]. Em linhas gerais, esses autores concordam que a redução na quantidade e tipo de boretos ocorreram devido ao aumento do teor de Fe nos revestimentos. Contudo, o papel do Fe ainda não está bem entendido. Ramasubbu et al. [2] explica que o $\mathrm{B}$ tem maior afinidade físico química com o Fe do que com o $\mathrm{Cr}$ mas, no presente estudo não foram identificados padrões de difração de boretos de $\mathrm{Fe}\left(\mathrm{FeB}\right.$ e $\mathrm{Fe}_{2} \mathrm{~B}$ ).

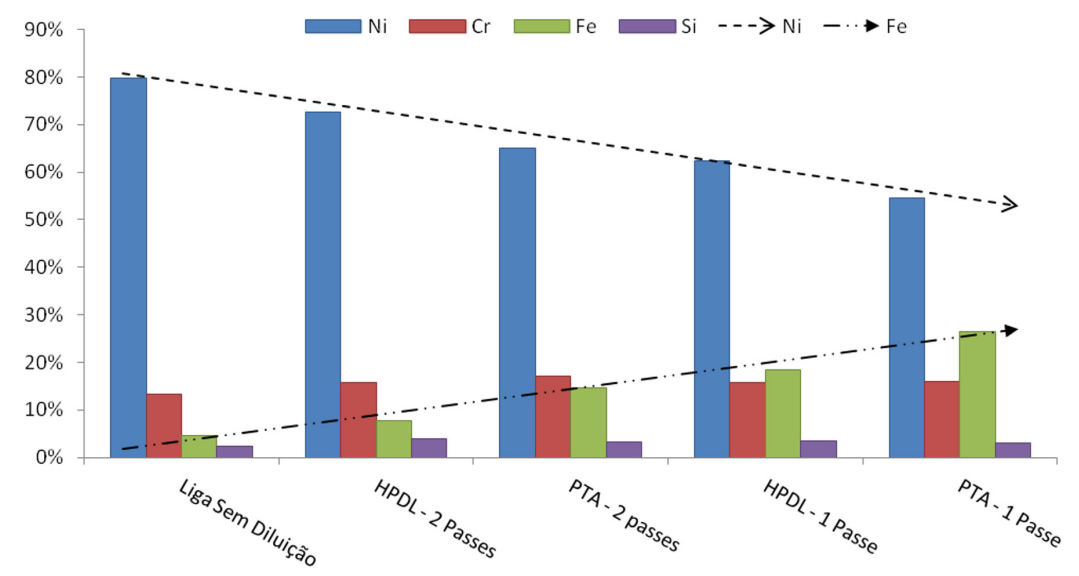

Figura 2. Efeito da diluição sobre composição química dos revestimentos.

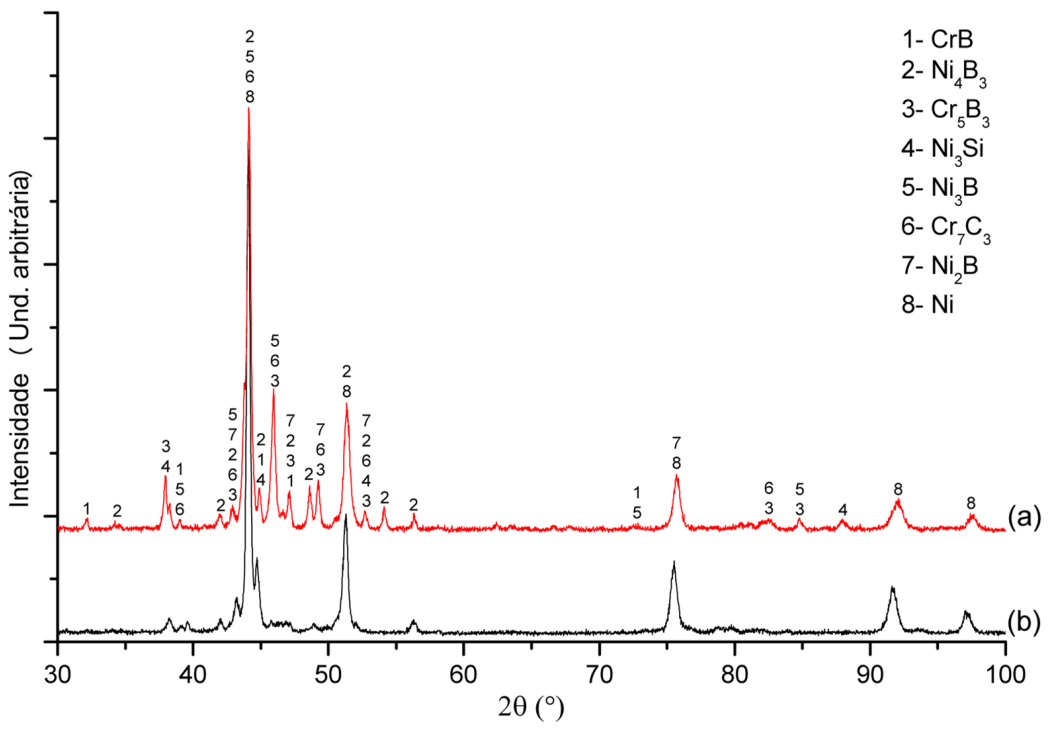

Figura 3. Efeito da diluição sobre a microestrutura dos revestimentos processados por PTA: (a) 14; e (b) 30\%. 
Neste trabalho, bem como, em outros realizados anteriormente $[1,6,8]$ com a liga NiCrSiBC, a identificação de fases foi realizada através de DRX e resultados de EDS auxiliaram na interpretação dos difratogramas. Entretanto, podem existir algumas limitações uma vez que, nesse caso as informações do DRX representam regiões macroscópicas que são correlacionados com os resultados de composição química obtida por EDS numa escala microscópica. $O$ processo de identificação pode ser particularmente difícil no caso de materiais multifásicas, onde os padrões difração de diferentes fases podem sobrepor, consequentemente causando incertezas (qual pico pertence a cada fase).

Misturas de fases com baixa simetria, como no caso da liga NiCrSiBC, normalmente são mais difíceis de identificar, devido ao grande número de picos. No caso dos revestimentos depositados por PTA e, principalmente, por HPDL a solubilidade estendida, forte textura e elevada tensão residual tornam a identificação de fases por DRX complexa. Com a utilização de métodos de otimização e refinamento, como Rietveld, grande parte dos problemas citados podem ser resolvidos. Hemmati [4], que realizou um estudo sobre diferentes métodos de caracterização da liga NiCrSiBC, sugere a técnica EBSD (Electron Backscatter Diffraction) como ferramenta alternativa de caracterização (segundo o autor, esta técnica representa um meio termo entre o DRX e o MET).

O forte impacto da diluição sobre a microestrutura dos revestimentos pode ser observado na seção transversal, junto à linha de fusão, Figura 4a. Esta região está exposta a um gradiente de composição mais significativo, o que resulta em uma intensa variação microestrutura, além da existência de uma faixa livre da formação de boretos (estrelares escuros). A liga inicialmente com uma estrutura hipereutética, em consequência de variações na composição química muda para uma estrutura eutética e na sequência hipoeutética, sendo esta alterações associadas ao perfil do teor de Fe [9], como mostrado no mapa de composição da Figura 4b.

Junto à linha de fusão, o elevado teor de Fe (Figura 4b) justifica o desenvolvimento da estrutura hipoeutética; ao se aumentar a distância da linha de fusão, em direção ao topo do revestimento, o teor de Fe diminui, favorecendo o desenvolvimento uma estrutura eutética. Maiores distancias da linha de fusão, reduzem o teor de Fe e contribuem para a formação de uma estrutura hipereutética, caracterizada pela intensa formação de carbonetos e boretos (agulhas de meio tom e estrelares escuros).

Além da variação da composição química, deve-se considerar a variação do parâmetro de solidificação G/R (G: gradiente de temperatura - R: velocidade de solidificação). Junto à linha de fusão $G$ é elevado e $R$ é baixa, logo a razão $G / R$ é elevada e a frente de solidificação é planar, o que significa que ocorre pouca segregação e os elementos de liga ficam retidos em solução sólida. À medida que a solidificação prossegue, $G$ reduz e $R$ aumenta e a segregação aumenta na frente de solidificação podendo se ter um crescimento dendrítico. A variação do parâmetro G/R ocorre rapidamente no início da solidificação, posterirormente permanece constante [10].

Micrografias do topo (distante da linha de fusão) dos revestimentos processados por PTA são apresentadas na Figura 5. Observa-se que ocorreram mudanças entre a microestrutura do primeiro e segundo cordão que podem
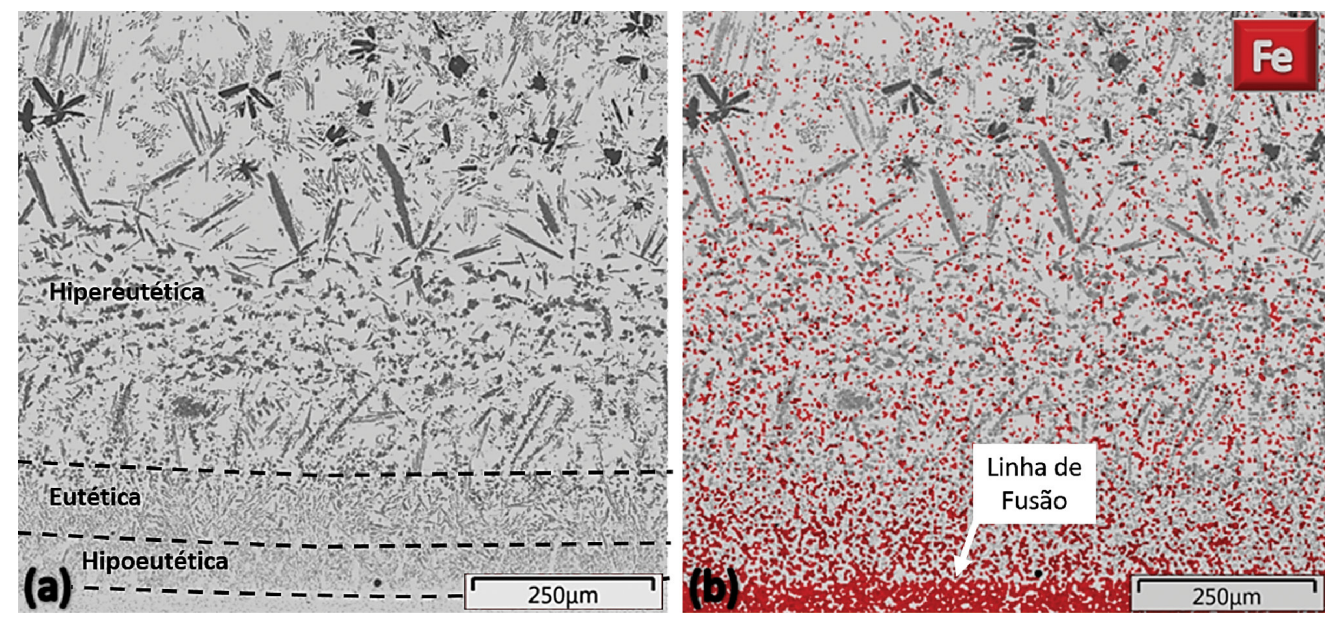

Figura 4. Linha de fusão do revestimento processado por PTA: (a) variação da microestrutura e (b) gradiente de Fe junto à linha de fusão. 
ser associadas a variação da diluição. Considerando a morfologia dos microconstituintes [7], a microestrutura do primeiro cordão é composta principalmente por carbonetos $\mathrm{Cr}_{7} \mathrm{C}_{3}$ (ripas, meio tom) e boretos $\mathrm{CrB}$ (regiões escuras), pontos 1 e 2 respectivamente indicados na Figura $5 a$. Já na microestrutura do segundo cordão, com menor diluição (Figura 5b), observa-se a intensa formação do boreto $\mathrm{Cr}_{5} \mathrm{~B}_{3}$ (ponto 3 - estrela), $\mathrm{Ni}_{3} \mathrm{~B}$ (estrutura ramificada) e carboneto $\mathrm{Cr}_{7} \mathrm{C}_{3}$ (ponto 4 -ripas).

Os resultados de EDS, apresentados na Tabela 6, corroboram com as conclusões de Rasmasubbu [2], que afirma que com o aumento da diluição, não apenas a composição química da matriz é alterada, mas também a composição química dos carbonetos e boretos. Isso fica claro comparando os teores de Fe do carboneto tipo $\mathrm{Cr}_{7} \mathrm{C}_{3}$ formado no primeiro cordão com maior diluição, EDS-1(22.1at\%Fe) da Tabela 6 (ponto 1 na Figura 5a), com um carboneto do mesmo tipo formado no segundo cordão com menor diluição, EDS-4 (11.1at\%Fe) (ponto 4 da Figura 5b).

Os boretos na microestrutura dos revestimentos processados por HPDL exibem uma morfologia diferenciada, como apresentado na Figura 6. Boretos, na forma de bloco ( $\mathrm{CrB}$ ), foram encontrados nas duas condições (1 cordão com $19 \%$ de diluição e 2 cordões com $4 \%$ de diluição), o aumento da diluição causou uma redução no tamanho dos boretos. Como os boretos são as primeiras fases a solidificar é razoável considerar que as alterações causadas na composição química do revestimento, ainda na poça de fusão, devido ao aumento do teor de Fe com a diluição, alteram a nucleação e a cinética de crescimento dos boretos [2].

Para efeito de correlação entre as técnicas de processamento, foram selecionados revestimentos processados por PTA e HPDL com diluições semelhantes, 14 e 19\%, Figura 7. As diferenças observadas quanto a granulometria e morfologia das partículas de solidificação hipereutética podem ser associadas principalmente a taxa de resfriamento imposta por cada um dos processos. Sendo que o maior refino dos revestimentos processados por HPDL pode ser explicado pelas maiores taxas de resfriamento. Entretanto, também se observam diferenças na formação do boretos $\mathrm{Cr}_{5} \mathrm{~B}_{3}$ (estrelares) nos revestimentos processados por PTA e CrB (cubos) para os revestimentos processados por HPDL, Figura 8. Esse comportamento pode ser explicado considerando a reação peritética $L+C r B \rightarrow C r_{5} B$ que ocorre a $1900{ }^{\circ} \mathrm{C}$ no diagrama binário $\mathrm{Cr}$ - $\mathrm{B}$. O crescimento do boreto $\mathrm{Cr}_{5} \mathrm{~B}_{3}$, sobre o $\mathrm{CrB}$, é controlado pela difusão de $\mathrm{B}$ e $\mathrm{Cr}$, assim pode-se associar a elevada taxa de resfriamento do processamento por HPDL com comprometimento do crescimento do boreto $\mathrm{Cr}_{5} \mathrm{~B}_{3}$. [7].

O impacto do processamento sobre microestrutura também se reflete na dureza dos revestimentos na Figura 9. Os revestimentos processados por HPDL, com 1 e 2 cordões, apresentaram maior dureza. Isso sugere que uma distribuição refinada e os boretos em forma de bloco também podem ter uma contribuição efetivos

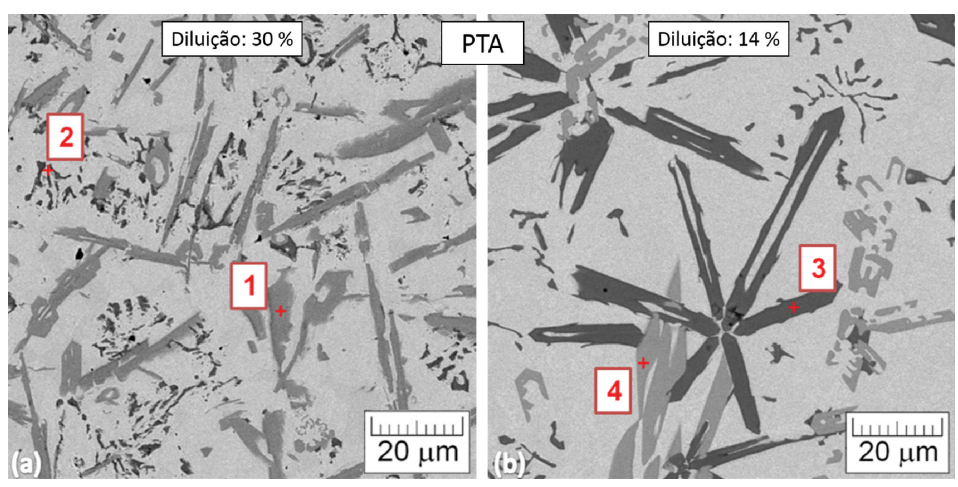

Figura 5. Efeito da diluição na microestrutura dos revestimentos processados por PTA: (a) 30\%; e (b) $14 \%$.

Tabela 6. Análise da composição química (EDS) referente aos pontos indicados na Figura 6 (\% peso).

\begin{tabular}{ccccccr}
\hline EDS & Ni & Fe & Cr & C & Si & B \\
1 & 5,9 & 22,1 & 50,4 & 5,8 & - & 15,8 \\
2 & 57,5 & 24,5 & 4,8 & 5,5 & 3,9 & 3,8 \\
3 & 19,9 & 5,8 & 58,5 & 4,4 & 0,7 & 17,7 \\
4 & 8,4 & 11,1 & 60,8 & 13,3 & - & 6,5 \\
\hline
\end{tabular}


no endurecimento, ou seja, dificultam mais a movimentação das discordâncias. Durezas semelhantes, para revestimentos processados com laser cladding foram encontrados por Hemmati [3] (750 HV para diluição de 5\% e 680 HV para diluição de 15\%). Durezas entre 430 e 480 HV para revestimentos depositados por PTA (1 cordão) foram reportadas por Gurumoorthy et al. [8] e Kesavan e Kamaraj [11,12] porém, esses autores não consideraram a diluição. Como não realizaram a sobreposição de cordões estima-se que a diluição seja semelhante a diluição encontrada no revestimento processado por PTA com único cordão (30\%).

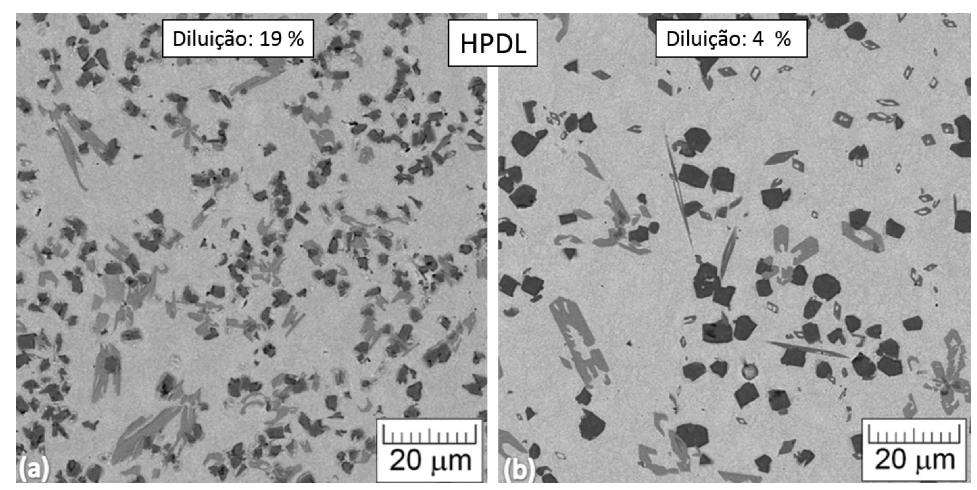

Figura 6. Efeito da diluição sobre microestrutura dos revestimentos processados por HPDL: (a) 19; e (b) 4\%.
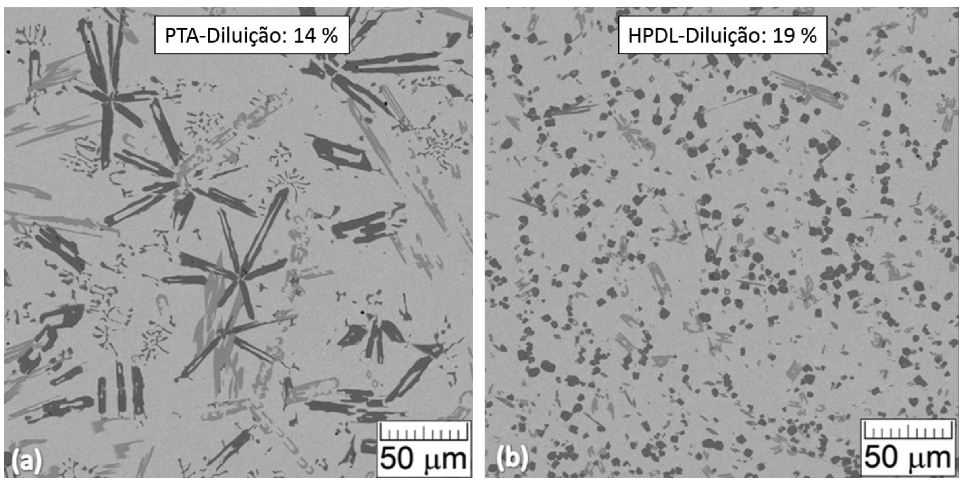

Figura 7. Impacto do processamento sobre microestrutura: (a) PTA - 14\% de diluição; e (b) HPDL - 19\%.

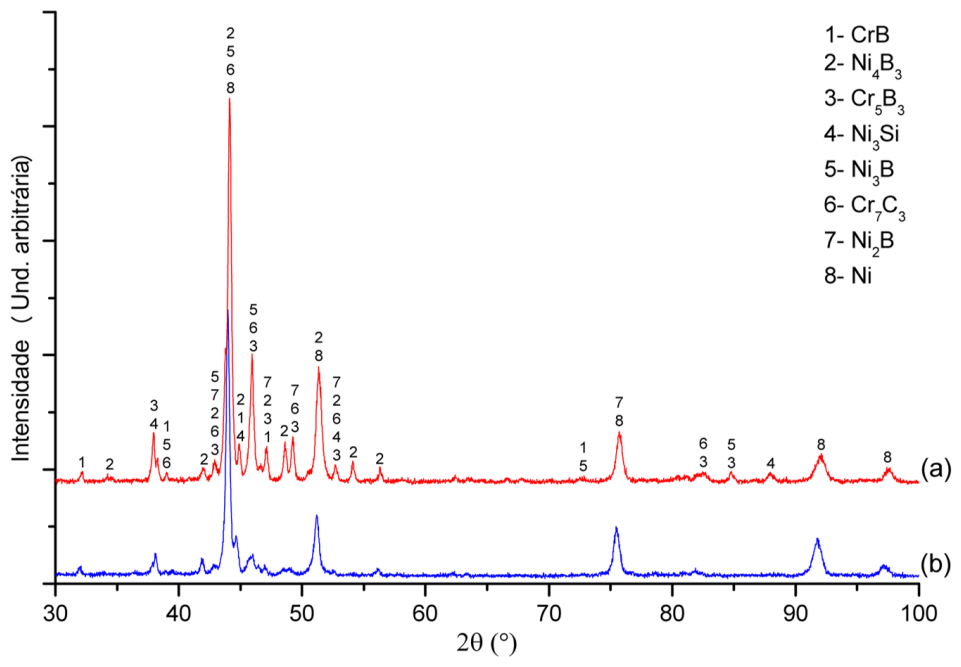

Figura 8. Comparação entre as fases formadas em um revestimento depositado por PTA com (a) $14 \%$ de diluição e outro depositado por HDPL com (b) $19 \%$. 


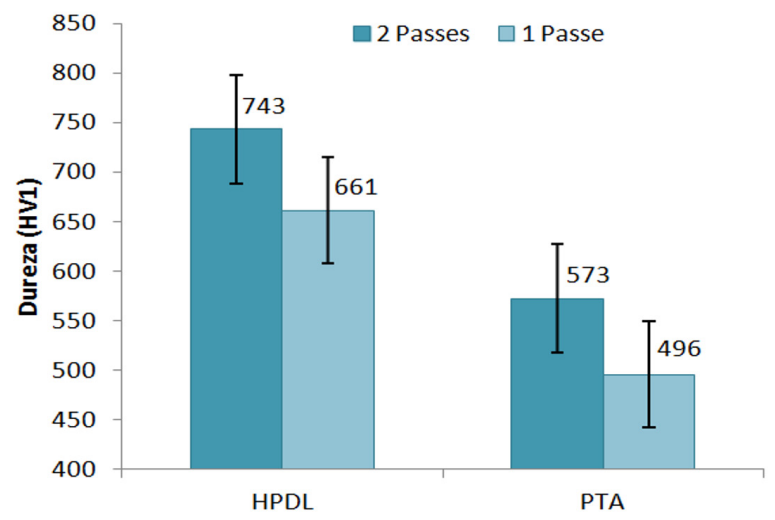

Figura 9. Dureza no topo dos revestimentos em função do processamento e do nível de diluição.

\section{Conclusões}

Neste estudo, a liga NiCrSiBC foi depositada por PTA e HPDL sobre AISI 304 com objetivo de avaliar o efeito da técnica de processamento na microestrutura dos revestimentos. Com base nos resultados apresentados conclui-se que:

- Para mesma técnica de processamento, a diluição com o substrato de AISI 304, em particular o teor de Fe altera significantemente a microestrutura e a dureza do revestimento;

- A distribuição, tamanho, morfologia e composição química de boretos, principalmente, é alterada com o aumento do teor de Fe proveniente do substrato;

- A taxa de resfriamento experimentada pelos depósitos durante a solidificação, também altera a morfologia de boretos e carbonetos no revestimento e consequentemente a dureza.

\section{Agradecimentos}

Os autores agradecem a CAPES-CNPq pelo financiamento, a empresa Oxipra Ltd pela deposição com HPDL e ao Instituto LACTEC pela utilização do MEV.

\section{Referências}

[1] Reinaldo PR, D'Oliveira ASCM. NiCrSiB Coatings deposited by plasma transferred arc on different steel substrates. Journal of Materials Engineering and Performance. 2013;22(2):590-597. http://dx.doi.org/10.1007/s11665-012-0271-7.

[2] Ramasubbu V, Chakraborty G, Albert SK, Bhaduri K. Effect of dilution on GTAW colmonoy 6 (AWS $\mathrm{NiCr}-\mathrm{C}$ ) hardface deposit made on $316 \mathrm{LN}$ stainless steel. Materials Science and Technology. 2011;27(2):573-580. http://dx.doi.org/10.1179/0 26708309X12526555493431.

[3] Hemmati I, Ocelík V, De Hosson JTM. Dilution effects in laser cladding of $\mathrm{Ni}-\mathrm{Cr}-\mathrm{B}-\mathrm{Si}-\mathrm{C}$ hardfacing alloys. Materials Letters. 2012;84:69-72. http://dx.doi.org/10.1016/j.matlet.2012.06.054.

[4] Hemmati I. Laser-deposited metallic coatings: processing, characterization, alloy development [doctor thesis]. Groningen: University of Groningen; 2013.

[5] Chandran S, Vinayak S. Effect of substrate dilution on corrosion of colmonoy- $6{ }^{\circledR}$ weld overlays in nitric acid. International Journal of Nuclear Energy Science and Technology. 2011;6(3):199-212. http://dx.doi.org/10.1504/IJNEST.2011.043395.

[6] Das CR, Albert SK, Bhaduri K, Nithya R. Effects of dilution on microstructure and wear behaviour of $\mathrm{NiCr}$ hardface deposits. Materials Science and Technology. 2007;23(7):771-779. http:// dx.doi.org/10.1179/174328407X185802.
[7] Hemmati I, Ocelík V, Csach K, De Hosson JTM. Microstructure and phase formation in a rapidly solidified laser-deposited $\mathrm{Ni}-\mathrm{Cr}-\mathrm{B}-\mathrm{Si}-\mathrm{C}$ hardfacing alloy. Metallurgical and Materials Transactions. A, Physical Metallurgy and Materials Science. 2014;45(2):878-892. http://dx.doi.org/10.1007/s11661-0132004-4.

[8] Gurumoorthy K, Kamaraj M, Rao KP, Rao S, Venugopal S. Microstructural aspects of plasma transferred arc surfaced $\mathrm{Ni}$-based hardfacing alloy. Materials Science and Engineering A. 2007;456(1-2):11-19. http://dx.doi.org/10.1016/j.msea.2006.12.121.

[9] Zhang H, Shi Y, Kutsuna M, Xu GJ. Laser cladding of Colmonoy 6 powder on AISI316L austenitic stainless steel. Nuclear Engineering and Design. 2010;240(10):2691-2696. http://dx.doi. org/10.1016/j.nucengdes.2010.05.040.

[10] Kou S. Welding metallurgy. 2. ed. New York: John Wiley \& Sons; 2002. http://dx.doi.org/10.1002/0471434027.

[11] Kesavan D, Kamaraj M. Influence of aging treatment on microstructure, wear and corrosion behavior of a nickel base hardfaced coating. Wear. 2011;272(1):7-17. http://dx.doi. org/10.1016/j.wear.2011.05.041.

[12] Kesavan D, Kamaraj M. The microstructure and high temperature wear performance of a nickel base hardfaced coating. Surface and Coatings Technology. 2010;204(24):4034-4043. http:// dx.doi.org/10.1016/j.surfcoat.2010.05.022. 